\title{
A THEOREM OF GREEN'S TYPE FOR FUNCTIONS OF TWO COMPLEX VARIABLES ${ }^{1}$
}

\section{STEFAN BERGMAN}

1. Domains with a distinguished boundary surface. Functions of the extended class. The real and imaginary parts of a function of $n$ complex variables are harmonic functions of $2 n$ real variables. Using Green's theorem one can transform Dirichlet integrals over a $2 n$ dimensional domain, say $D$, into integrals over the $(2 n-1)$-dimensional boundary of $D$. Using this procedure (and exploiting some further properties of harmonic functions) one obtains generalizations of the Cauchy formula (see Bergman [1], ${ }^{2}$ Bochner-Martin [5], Martinelli [8]), certain generalizations of Nevanlinna's theory of meromorphic functions, etc. Recently, applying Green's transformations, Garabedian obtained an important generalization to the case of several variables of formulas connecting Green's and Neumann's functions with the kernel function [6]. Further, Garabedian and Spencer [7] showed that these methods can be extended to the theory of "analytic tensors" defined on certain Kähler manifolds. On the other hand, the real and imaginary parts of functions of $\boldsymbol{n}$ complex variables, $n>1$, represent a very special class of harmonic functions and by the above theorems the possibilities of using theorems of Green's type are in no way exhausted. One can apply, in this special case, reduction of Green's type of $k$-dimensional integrals, for $k>n$, repeatedly.

In the present paper we shall discuss an example of such a procedure in the case of functions of two complex variables. In order to explain our approach it will be useful to discuss more in detail the geometrical situation which we meet in this theory. For the sake of simplicity, we shall limit ourselves to the case $n=2$ and consider only a very special type of domains. ${ }^{3}$ As has been indicated in $[3 ; 2$, chap. I], the geometry of the space of functions of two complex variables differs in many respects from the geometry of four-dimensional Euclidean space. One of the reasons for this situation is the fact that analytic surfaces and segments of these surfaces take over to a certain extent the role of points in Euclidean geometry. For in-

Received by the editors April 2, 1952.

1 This paper was done under Contract N5ori 76/XVI NR 043068.

In particular, see p. 389.

In the general case it is convenient to use a certain symbolism (e.g., that of Cartan's "exterior forms"). 
stance, a function $f$ of two complex variables assumes a constant value only in an analytic surface and $f$ is itself an analytic function of one complex variable in every analytic surface. In developing the theory of two complex variables it is useful to pay special attention to domains which are bounded by a finite number of segments of analytic hypersurfaces (one-parameter families of analytic surfaces). On the boundary of such a domain lies the distinguished boundary surface (the totality of the intersections of at least two segments of the analytic hypersurfaces belonging to the boundary). The distinguished boundary surface plays in many respects a role similar to that of the boundary curve in the case of the theory of functions of one variable. For instance, the maximum of the absolute value of a function is assumed not only on the boundary but even at a point of the distinguished boundary surface. There exists an analogue of the Cauchy formula representing the function $f\left(z_{1}, z_{2}\right), z_{k}=x_{2 k-1}+i x_{2 k}$, $k=1,2$, inside the domain in terms of the values on the distinguished boundary surface. There is, however, an important difference between the situation in one and two variables. If a real, continuously differentiable function is prescribed on the distinguished boundary surface, there does not in general exist a $B$-harmonic function (real part of an analytic function of two complex variables) which assumes on the distinguished boundary surface the prescribed values. In order to develop techniques along lines similar to those used for functions of one variable (for instance, to be able to apply potentialtheoretic methods), it is useful to introduce in addition to $B$-harmonic functions a larger class of functions, those of the so-called extended class $[3 ; 4]$. They include the $B$-harmonic functions; on the other hand, this class is sufficiently large so that the boundary value problem with the values prescribed on the distinguished boundary surface has always a unique solution. The extended class of functions depends upon the domain. The doubly-harmonic functions, i.e., functions $u$ which satisfy

$$
\left(\partial^{2} u / \partial x_{2 k-1}^{2}\right)+\left(\partial^{2} u / \partial x_{2 k}^{2}\right)=0, \quad k=1,2,
$$

form the extended class in the case of a bicylinder.

In order to use potential-theoretic methods it is necessary often to derive theorems of Green's type for functions of the extended class. A theorem of this type will be proved in the next section.

In the following we shall consider a special subclass of domains of the above type. (The method used here can be easily extended to more general domains with distinguished boundary surface.) These domains can be defined as follows. We consider the $\zeta_{1}, \zeta_{2}$-space where 


$$
\zeta_{k}=\xi_{2 k-1}+i \xi_{2 k}=\rho_{k} e^{i \lambda_{k}}, \quad k=1,2 .
$$

Let

$$
h\left(\zeta_{1}, \zeta_{2}\right) \equiv h^{(1)}\left(\rho_{1}, \lambda_{1} ; \rho_{2}, \lambda_{2}\right)+i h^{(2)}\left(\rho_{1}, \lambda_{1} ; \rho_{2}, \lambda_{2}\right)
$$

be an analytic function of $\zeta_{1}, \zeta_{2}$ in $\left[1-\epsilon<\rho_{1}<1+\epsilon, \rho_{2} \leqq 1\right]$, which for fixed $\zeta_{2}$ is a single-valued and univalent function of $\zeta_{1}$, for $1-\epsilon<\rho_{1}$ $<1+\epsilon$. Let $z_{k}=x_{2 k-1}+i x_{2 k}, k=1,2$, denote two complex variables, and let $p\left(\zeta_{2}^{0}\right),\left|\zeta_{2}^{0}\right| \leqq 1$ be the curve

$$
\left[z_{1}=h\left(e^{i \lambda_{1}}, \zeta_{2}^{0}\right), 0 \leqq \lambda_{1} \leqq 2 \pi, z_{2}=\zeta_{2}^{0}\right] .
$$

By the definition of $h\left(\zeta_{1}, \zeta_{2}\right), p\left(\zeta_{2}^{0}\right)$ will be a simple closed curve lying in the plane $z_{2}=\zeta_{2}^{0}$ of the $z_{1}, z_{2}$-space. The interior of $p\left(\zeta_{2}^{0}\right)$ in this plane we shall denote by $P\left(\zeta_{2}^{0}\right)$. We note that $p\left(\zeta_{2}\right)$ varies continuously as $\zeta_{2}$ varies, $\left|\zeta_{2}\right| \leqq 1$. The domain $D$ of the $z_{1}, z_{2}$-space which we shall consider in the following is defined by

$$
D=\sum_{\left|\zeta_{2}\right|<1} P\left(\zeta_{2}\right)
$$

The boundary of $D$ consists of two segments of analytic hypersurfaces:

$$
c=\sum_{0 \leqq \lambda_{2} \leqq 2 \pi} P\left(e^{i \lambda_{2}}\right) \text { and } d=\sum_{0 \leqq \lambda_{1} \leqq 2 \pi} B_{1}\left(e^{i \lambda_{1}}\right)
$$

where

$$
B_{1}\left(e^{i \lambda_{1}}\right)=\left[z_{1}=h\left(e^{i \lambda_{1}}, \zeta_{2}\right), z_{2}=\zeta_{2} ;\left|\zeta_{2}\right| \leqq 1\right] .
$$

We note that $d$ can be represented also in the form

$$
d=\sum_{\left|\zeta_{2}\right| \vdots_{1}} p\left(\zeta_{2}\right)
$$

Thus the domain $D$ is defined in the $z_{1}, z_{2}$-space by the fact that its boundary $c+d$ is given by a mapping of the boundary of the unit bicylinder in the $\zeta_{1}, \zeta_{2}$-space.

$$
S=\sum_{0 \leqq \lambda_{2} \leqq 2 \pi} \rho\left(e^{i \lambda_{2}}\right)
$$

is the distinguished boundary surface of $D$.

A function $H\left(x_{1}, x_{2}, x_{3}, x_{4}\right) \equiv H\left(z_{1}, z_{2}\right)$ of the extended class in $D$ (which class we shall denote by $E(D)$ ) is defined by the following two properties.

$1^{\circ}$. For every fixed $z_{2}^{0},\left|z_{2}^{0}\right| \leqq 1, H\left(z_{1}, z_{2}^{0}\right)$ is harmonic in $x_{1}, x_{2}$ for $z_{1} \in P\left(\zeta_{2}^{0}\right)$, and continuously differentiable in $\bar{P}\left(\zeta_{2}^{0}\right), z_{2}^{0}=\zeta_{2}^{0}, \bar{P}=P+p$. 
$2^{\circ}$. For every fixed $\zeta_{1},\left|\zeta_{1}\right|=1, H\left(z_{1}, z_{2}\right)=H\left[h\left(\zeta_{1}, \zeta_{2}\right), \zeta_{2}\right]$ is a harmonic function of $\xi_{3}, \xi_{4}$ for $\left|\zeta_{2}\right|<1$ and continuously differentiable for $\left|\zeta_{2}\right| \leqq 1, \zeta_{2}=\xi_{8}+i \xi_{4}$.

2. A theorem of Green's type. Let $H^{(v)}\left(x_{1}, x_{2}, x_{3}, x_{4}\right), \nu=1,2$, be two functions of the class $E(D)$. By the definition of $D$ we may consider $H^{(\nu)}$ as a function of $\zeta_{1}, \zeta_{2}$ in the domain

$$
R=\left[1-\epsilon<\rho_{1} \leqq 1, \rho_{2} \leqq 1\right] .
$$

We may consider an arbitrary function $Q\left(z_{1}, z_{2}\right)$ as a function of $\zeta_{1}, \zeta_{2}$ in $R$. We shall indicate this by adding *. Thus

$$
\left(Q\left(z_{1}, z_{2}\right)\right)^{*} \equiv Q\left(h\left(\zeta_{1}, \zeta_{2}\right), \zeta_{2}\right) .
$$

Using this notation we define

$$
\begin{aligned}
G^{(\nu)}\left(\zeta_{1}, \zeta_{2}\right)=\left(B^{(\nu)}\left(z_{1}, z_{2}\right)\right)^{*}, & \nu=1,2, \\
L_{k}^{(\nu)}\left(\zeta_{1}, \zeta_{2}\right)=\left(\frac{\partial H^{(\nu)}\left(z_{1}, z_{2}\right)}{\partial x_{k}}\right)^{*}, & \nu=1,2 ; k=1,2 .
\end{aligned}
$$

Further, let

$$
\begin{aligned}
S\left(G^{(v)}\right) & =G^{(p)}-\sum_{k=1}^{2} L_{k}^{(p)} h^{(k)}, \\
\nabla & \equiv\left(\frac{\partial}{\partial \xi_{3}}, \frac{\partial}{\partial \xi_{4}}\right) .
\end{aligned}
$$

We introduce the following integrals:

$$
\begin{aligned}
F\left(H^{(1)}, H^{(2)} ; D\right) & \\
= & \iiint \int_{D} \sum_{k=1}^{2} \sum_{m=3}^{4}\left(\frac{\partial^{2} H^{(1)}}{\partial x_{k} \partial x_{m}} \frac{\partial^{2} H^{(2)}}{\partial x_{k} \partial x_{m}}\right) d x_{1} d x_{2} d x_{2} d x_{4} ; \\
T\left(H^{(1)}, H^{(2)} ; d\right) & =-\int_{\lambda_{1}=0}^{2 \pi} \iint_{B}\left[\left(\nabla G^{(1)}-\sum_{k=1}^{2} L_{k}^{(1)} \nabla h^{(k)}\right)\right. \\
& \frac{\partial}{\partial \rho_{1}}\left(\sum_{n=1}^{2}\left(h^{(1)} \nabla L_{v}^{(2)}\right)\right) \\
& \left.+\sum_{k=1}^{2}\left(\nabla h^{(k)} \cdot \nabla L_{k}^{(1)}\right) \frac{\partial S\left(G^{(2)}\right)}{\partial \rho_{1}}\right] d \lambda_{1} d \omega_{2} ;
\end{aligned}
$$




$$
\begin{aligned}
t\left(H^{(1)}, H^{(2)} ; d\right) & \\
= & -\int_{\lambda_{1}=0}^{2 \pi} \iint_{B}\left[S\left(G^{(1)}\right) \frac{\partial}{\partial \rho_{1}}\left(\sum_{v=1}^{2} \nabla L_{\nu}^{(2)} \cdot \nabla h^{(\nu)}\right)\right. \\
& \left.+\sum_{k=1}^{2} h^{(k)} \nabla L_{k}^{(1)} \cdot \frac{\partial}{\partial \rho_{1}}\left(\nabla G^{(2)}-\sum_{v=1}^{2} L_{v}^{(2)} \nabla h^{(v)}\right)\right] d \lambda_{1} d \omega_{2} ;
\end{aligned}
$$

$D\left(H^{(1)}, H^{(2)} ; S\right)$

$$
=\int_{\lambda_{1}=0}^{2 \pi} \int_{\lambda_{2}=0}^{2 \pi}\left(\frac{\partial G^{(1)}}{\partial \rho_{2}}-\sum_{k=1}^{2} L_{k}^{(1)} \frac{\partial h^{(k)}}{\partial \rho_{2}}\right) \frac{\partial S\left(G^{(2)}\right)}{\partial \rho_{1}} d \lambda_{1} d \lambda_{2} ;
$$

$$
d\left(H^{(1)}, H^{(2)}: S\right)
$$

$$
=\int_{\lambda_{1}=0}^{2 \pi} \int_{\lambda_{2}=0}^{2 \pi} S\left(G^{(1)}\right) \frac{\partial}{\partial \rho_{1}}\left[\frac{\partial G^{(2)}}{\partial \rho_{2}}-\sum_{v=1}^{2} L_{\nu}^{(2)} \frac{\partial h^{(v)}}{\partial \rho_{2}}\right] d \lambda_{1} d \lambda_{2} .
$$

Here $B=\left[\left|z_{2}\right|<1\right]$.

ThEOREM. Let $H^{(1)}$ and $H^{(2)}$ be two functions of the extended class in the domain $D$. Then

$$
\begin{aligned}
F\left(H_{1}, H_{2} ; D\right) & =D\left(H^{(1)}, H^{(2)} ; S\right)+T\left(H^{(1)}, H^{(2)} ; d\right) \\
& =d\left(H^{(1)}, H^{(2)} ; S\right)+t\left(H^{(1)}, H^{(2)} ; d\right) .
\end{aligned}
$$

Proof. Since $H^{(2)}$ for a fixed $x_{8}, x_{4}\left(x_{3}^{2}+x_{4}^{2} \leqq 1\right)$ is a harmonic function of $x_{1}, x_{2}$ in $P\left(z_{2}\right)$, continuously differentiable in $P\left(z_{2}\right)+p\left(z_{2}\right)$,

$$
F\left(H^{(1)}, H^{(2)} ; D\right)
$$

$$
=-\iint_{B}\left(\int_{p\left(z_{2}\right)} \sum_{m=3}^{4} \frac{\partial H^{(1)}}{\partial x_{m}} \frac{\partial}{\partial n_{1}}\left(\frac{\partial H^{(2)}}{\partial x_{m}}\right) d s_{1}\right) d x_{8} d x_{4}
$$

where $n_{1}$ is the interior normal to $p\left(\zeta_{2}\right)$ and $d s_{1}$ the line element of $p\left(\zeta_{2}\right)$. Since $h\left(\zeta_{1}, \zeta_{2}\right)$ for fixed $\zeta_{2},\left|\zeta_{2}\right|<1,1-\epsilon<\left|\zeta_{1}\right| \leqq 1$, is an analytic function of $\zeta_{1}$,

$$
\frac{\partial}{\partial n_{1}}=\left|\frac{\partial h\left(\zeta_{1}, \zeta_{2}\right)}{\partial \zeta_{1}}\right|^{-1} \frac{\partial}{\partial \rho_{1}}, d s_{1}=\left|\frac{\partial h\left(\zeta_{1}, \zeta_{2}\right)}{\partial \zeta_{1}}\right| d \lambda_{1}
$$

and therefore

$$
\begin{aligned}
& F\left(H^{(1)}, H^{(2)} ; D\right) \\
& =-\iint_{B} \int_{\lambda_{1}=0}^{2 \pi}\left[\sum_{m=3}^{L}\left(\frac{\partial H^{(1)}}{\partial x_{m}}\right)^{*} \frac{\partial}{\partial \rho_{1}}\left[\left(\frac{\partial H^{(2)}}{\partial x_{m}}\right)^{*}\right]\right] d \lambda_{1} d \xi_{2} d \xi_{4} .
\end{aligned}
$$

In the following the subscript $m$ after a function will indicate a partial derivative with respect to $\xi_{m}$, e.g., $G_{m}^{(v)} \equiv \partial G^{(v)} / \partial \xi_{m}$. Obviously, 


$$
\left(\frac{\partial H^{(v)}}{\partial x_{m}}\right)^{*}=G_{m}^{(v)}-\sum_{k=1}^{2} L_{k}^{(v)} h_{m}^{(k)}, \quad \nu=1,2 ; m=3,4
$$

Thus,

$$
\begin{aligned}
F\left(H^{(1)}, H^{(2)} ; D\right) & \\
= & -\int_{\lambda_{1}=0}^{2 \pi} \iint_{B} \sum_{m=-1}^{4}\left[\left(G_{m}^{(1)}-\sum_{k=1}^{2} L_{k}^{(1)} h_{m}^{(k)}\right)\right. \\
& \left.\cdot\left(\frac{\partial}{\partial \rho_{1}}\left(G_{m}^{(2)}-\sum_{m=1}^{2} L_{v}^{(2)} h_{m}^{(v)}\right)\right)\right] d \xi_{3} d \xi_{1} d \lambda_{1} \\
= & -\int_{\lambda_{1}=0}^{2 \pi} \iint_{B}\left(\sum _ { m = 3 } ^ { 4 } \left[\left(\frac{\partial G^{(2)}}{\partial \rho_{1}}\right) G_{m}^{(1)}-\sum_{m=1}^{2}\left(\frac{\partial L_{v}^{(2)}}{\partial \rho_{1}} h_{m}^{(v)} G_{m}^{(1)}\right.\right.\right. \\
& \left.+L_{v}^{(2)}\left(\frac{\partial h^{(v)}}{\partial \rho_{1}}\right) G_{m}^{(1)}\right)-\sum_{k=1}^{2} L_{k}^{(1)}\left(\frac{\partial G^{(2)}}{\partial \rho_{1}}\right)_{m}^{(k)} h_{m}^{(2)} \\
& +\sum_{v=1}^{2} \sum_{k=1}^{2}\left(L_{k}^{(1)} \frac{\partial L_{v}^{(2)}}{\partial \rho_{1}} h_{m}^{(v)} h_{m}^{(k)}\right. \\
& \left.\left.\left.+L_{k}^{(1)} L_{v}^{(2)}\left(\frac{\partial h^{(v)}}{\partial \rho_{1}}\right)_{m} h_{m}^{(k)}\right)\right]\right) d \xi_{3} d \xi_{1} d \lambda_{1} .
\end{aligned}
$$

If we assume that $V$ is a harmonic function of $\xi_{3}$ and $\xi_{4}$ in $B$ $=\left[\left(\xi_{3}^{2}+\xi_{4}^{2}\right)<1\right]$ and $T, A$, and $V$ are continuously differentiable in $\bar{B}$, then

$$
\begin{aligned}
\iint_{B} \sum_{m=3}^{4} T A_{m} V_{m} d \xi_{3} d \xi_{4} \equiv & \iint_{B} T \nabla A \cdot \nabla V d \xi_{3} d \xi_{4} \\
= & -\iint_{b} T A \frac{\dot{\partial} V}{\partial n_{2}} d s_{2} \\
& -\iint_{B} A \nabla T \cdot \nabla V d \xi_{3} d \xi_{4}
\end{aligned}
$$

where $n_{2}$ denotes the interior normal to $b=\left[\xi_{3}^{2}+\xi_{4}^{2}=1\right]$ and $d s_{2}$ the line element of $b$.

According to our assumptions $G^{(1)}, h^{(k)}, k=1,2$, are harmonic functions of $\xi_{3}, \xi_{4}$. Substituting these functions for $V$, replacing $\partial / \partial n_{2}$ by $\partial / \partial \rho_{2}, d s_{2}$ by $d \lambda_{2}$, and rearranging the terms, we obtain (2.11) from (2.18).

Similarly, using the fact that $\partial G^{(2)} / \partial \rho_{1}, \partial h^{(k)} / \partial \rho_{1}, k=1,2$, are also harmonic functions of $\xi_{3}, \xi_{4}$, we may substitute these quantities for 
$V$ into (2.19). In the same way as before we obtain (2.12) from (2.18).

3. Some applications of the theorem of $\$ 2$ to the theory of functions of two complex variables. The introduction of the operators permits us obviously to generalize a number of procedures in the theory of functions of one complex variable to the case of two complex variables. It is, for instance, clear that

$$
T(H, H ; d)+D(H, H ; S)=t(H, H ; d)+d(H, H ; S)
$$

is an increasing functional of the domain, i.e., if we have two domains of the structure described in $\$ 1$ such that one domain lies inside the other, then (3.1) for a (nonconstant) biharmonic function $B$ is larger than the corresponding expression for the smaller domain.

REMARK. Since the real part $B$ of a function of two complex variables satisfies the equations

$$
\frac{\partial^{2} B}{\partial x_{1} \partial x_{3}}=-\frac{\partial^{2} B}{\partial x_{2} \partial x_{4}}, \quad \frac{\partial^{2} B}{\partial x_{2} \partial x_{3}}=\frac{\partial^{2} B}{\partial x_{1} \partial x_{4}},
$$

we see that $F\left(B^{(1)}, B^{(2)} ; D\right)$ can be simplified by substituting

$$
\begin{aligned}
\frac{1}{2} \sum_{m=1}^{2} \sum_{n=3}^{4} \frac{\partial^{2} B^{(1)}}{\partial x_{m} \partial x_{n}} \frac{\partial^{2} B^{(2)}}{\partial x_{m} \partial x_{n}} & =\sum_{n=3}^{4} \frac{\partial^{2} B^{(1)}}{\partial x_{1} \partial x_{n}} \frac{\partial^{2} B^{(2)}}{\partial x_{1} \partial x_{n}} \\
& =\sum_{m=1}^{2} \frac{\partial^{2} B^{(1)}}{\partial x_{m} \partial x_{3}} \frac{\partial^{2} B^{(2)}}{\partial x_{m} \partial x_{3}}=\cdots
\end{aligned}
$$

It is further quite simple to obtain quantitative inequalities for the functionals (3.1). As an example for the application of this procedure we shall formulate the following:

LEMMA. Let a function

$$
f=\sum_{m, n=1}^{\infty} a_{m n} z_{1}^{m} z_{2}^{n}
$$

of two complex variables be regular in a (closed) domain D. Let us further assume that a Reinhardt circular domain

$$
R=\left[\left|z_{1}\right|^{2}<K\left(\left|z_{2}\right|^{2}\right),\left|z_{2}\right|^{2}<\alpha^{2}\right]
$$

lies inside of $D$. Then

$$
T(H, H ; d)+D(H, H ; S)
$$

$$
\geqq 2 \pi^{2} \sum_{m, n=0}^{\infty}(m+1)(n+1)^{2}\left|a_{m+1, n+1}\right|^{2} \int_{\tau=0}^{\alpha} \tau^{2 n}\left[K\left(\tau^{2}\right)\right]^{m+1} d\left(\tau^{2}\right) .
$$


Proof. A function $f$ of two complex variables can be represented in $R$ by the power series (3.4) which converges in $R$ uniformly and absolutely. Due to the orthogonality of powers $z_{1}^{m}, z_{2}^{n}$, in $R$,

$$
\begin{aligned}
& \iiint \int_{R}\left|\frac{\partial^{2} f}{\partial z_{1} \partial z_{2}}\right|^{2} d \omega \\
& =\sum_{m, n=0}^{\infty}(m+1)^{2}(n+1)^{2}\left|a_{m+1, n+1}\right|^{2} \iiint \int_{R}\left|z_{1}\right|^{2 m}\left|z_{2}\right|^{2 n} d \omega \\
& =\pi^{2} \sum_{m, n=0}^{\infty}(m+1) .(n+1)^{2}\left|a_{m+1, n+1}\right|^{2} \int_{\tau=0}^{\alpha} \tau^{2 n}\left(K\left(\tau^{2}\right)\right)^{m+1} d\left(\tau^{2}\right) .
\end{aligned}
$$

But, using formula (3.2) we see that if $f=H+i B_{2}$,

$$
\left|\frac{\partial^{2} f}{\partial z_{1} \partial z_{2}}\right|^{2}=\frac{1}{2} \sum_{m=1}^{2} \sum_{n=8}^{4}\left(\frac{\partial^{2} H}{\partial x_{m} \partial x_{n}}\right)^{2} .
$$

\section{BIBLIOGRAPHY}

1. S. Bergman, Über uneigentliche Flächenintegrale in der Theorie der analytischen Funktionen von zwei komplexen Veränderlichen, Revista de Ciencias, Universidad Nactional Mayor de San Marcos, Lima, Peru, No. 438, pp. 675-682; No. 439, pp. 131-140; No. 44, 1941-42, pp. 377-394.

2. - Sur les fonctions orthogonales de plusieur variables complexes avec les applications $d$ la theorie des fonctions analytiques, Interscience Publishers, 1941, and Mémorial des Sciences Mathématiques, vol. 106, Paris, 1947.

3. - Geometric and potentialtheoretical methods in the theory of functions of several complex variables, Proceedings of the International Congress of Mathematicians, Cambridge, 1950, vol. 2, pp. 165-173.

4. - Functions of the extended class in the theory of functions of several complex variables, Trans. Amer. Math. Soc. vol. 63 (1948) pp. 423-447.

5. S. Bochner and W. T. Martin, Several complex variables, Princeton Press, 1948.

6. P. Garabedian, $A$ new formalism for functions of several complex variables, Journal d'Analyse Mathématique vol. 1 (1951) pp. 59-80.

7. P. Garabedian and D. C. Spencer, A complex tensor calculus for Kahler manifolds, to appear in Acta Mathematica.

8. E. Martinelli, Alcuni teoremi integrali per le funzioni analitiche di pu variabili complesse, Memorie R. Acc. d'Italia vol. 9 (1938) pp. 269-283.

\section{HARVARD UNIVERSITY}

